\title{
Gamma knife radiosurgery of recurrent central neurocytomas: a preliminary report
}

\author{
A Bertalanffy, K Roessler, W Dietrich, M Aichholzer, D Prayer, A Ertl, K Kitz
}

\begin{abstract}
Objectives-A series of three recurrent central neurocytomas treated by gamma knife radiosurgery (GKRS), which were initially totally resected, are described. Up to now, no reports exist on this treatment modality for this rare tumour entity. Methods-Three male patients, aged between 20 and 25 years, presented with large intraventricular tumours. Total tumour removal was achieved by a single surgical procedure (one patient) or two operations (two patients). Neuropathological investigation showed a central neurocytoma, immunohistochemically all three tumours expressed a neuronal antigenetic profile typical for neurocytomas, and the MIB-1 proliferation index ranged from $2.4 \%$ to $8.7 \%$. Each patient experienced a tumour recurrence after 5 to 6 years. The recurrence was multifocal in two and a singular tumour mass in one patient. Gamma knife radiosurgery was performed. The tumours were enclosed within the $30 \%$ to $60 \%$ isodoseline, and delivered a tumour marginal dose of 9.6 to 16 Gy. During the follow up period, the patients were tested clinically and the volume of the tumours was measured on MRI.
\end{abstract}

Results-Within follow up periods of 1 to 5 years, control MRI showed a significant decrease of the tumour mass in all cases. None of the patients developed new neurological symptoms after GKRS. Two patients returned to work in their previous employment, whereas one patient remained permanently disabled due to a pre-existing visual impairment and abducens palsy.

Conclusion-GKRS proved to be a useful tool in the treatment of recurrent central neurocytomas. Tumour control and even tumour shrinkage can be achieved with a single procedure and a low risk of morbidity.

(F Neurol Neurosurg Psychiatry 2001;70:489-493)

Keywords: central neurocytoma; recurrence; gamma knife

Department of Radiology, Division of Neuroradiology

D Prayer

Correspondence to: Dr A Bertalanffy alexander.bertalanffy@ univie.ac.at

Received 17 April 2000 and in revised form

16 August 2000

Accepted 27 November 2000 or without extension to the third ventricle, and they have also been reported to occur sporadically at other locations. ${ }^{5-10}$ Usually they present with a benign clinical course, although recurrences and malignant forms have been described. ${ }^{411-13}$

Microsurgical resection is commonly regarded as the treatment of choice. ${ }^{14-17}$ As total resection is not always possible, the question concerning a safe and effective adjunctive treatment option of residual tumours and recurrences remains topical. Only very few reports exist about chemotherapy. ${ }^{15}{ }^{18}$ Fractionated (whole brain or local) radiation therapy is still controversial, although many authors suggest this treatment option after total resection as well as after subtotal or partial resection. ${ }^{14-171920}$ Some authors report on tumour shrinkage and even tumour disappearance after conventional radiation therapy, delivering 48 Gy to $61 \mathrm{~Gy}$ by 180 cGy to 200 cGy fractions. ${ }^{14} 151719$

Gamma knife radiosurgery was pioneered in Sweden in $1968 .^{21}$ The mechanism is a precise, single session, closed skull destruction of an intracranial target by ionising beams of radiation. ${ }^{22}$ Especially within the past 10 years it has gained increasing importance in the treatment of malignant as well as benign tumours. By contrast with open surgery, the aim of GKRS in benign tumours such as acoustic neurinomas or meningeomas is not total tumour removal, but long term tumour control. ${ }^{23}$

This paper retrospectively summarises our experiences with GKRS in the treatment of recurrent central neurocytomas. Special attention was given to the efficacy of radiosurgery on changes in tumour volume and on postradiosurgical morbidity.

To our knowledge, this constitutes the first report on the use of GKRS in central neurocytomas.

Material and methods

Between 1987 and 1991 we encountered three male patients, aged between 19 and 25 years, who all became symptomatic with signs of increased intracranial pressure (table 1). In addition we found hemihypaesthesia, abducens palsy, and visual impairment. The neuroradiological investigation disclosed a hydrocephalus in all patients, the tumours had strong contrast enhancement and cystic parts. By means of one to two surgical procedures total resection of tumours was achieved in all three patients, documented by postoperative CT. Neuropathological investigation classified the tumours as central neurocytomas and showed immunopositivity for neuron specific enolase 
Table 1 Patient characteristics and radiosurgical technique

\begin{tabular}{lllllll}
\hline & Sex & Age & Surgeries (n) & $\begin{array}{l}\text { Marginal } \\
\text { dose (Gy) }\end{array}$ & Isodose line (\%) & Isocentres (n) \\
\hline Patient 1 & Male & 23 & 2 & 12.5 & 50 & 3 \\
Patient 2 & Male & 19 & 2 & $9.6-16$ & $30-50$ & 17 \\
Patient 3 & Male & 25 & 1 & 13 & 60 & 12 \\
\hline
\end{tabular}

(NSE) and synaptophysin (SYN) but immunonegativity for glial fibrillary acidic protein (GFAP), S 100 protein (S 100), and neurofilament protein (NFP) in each tumour. The MIB-1 proliferation indices were $2.4 \%, 7 \%$, and $8.7 \%$.

Five to 6 years after the last surgical procedure, there were tumour recurrences in both lateral ventricles, as a singular tumour nodule in one patient, and multifocal in two. An additional tumour nodule in the third ventricle was seen in one patient. Before radiosurgery, two patients had shown no neurological deficit. One patient had an abducens palsy and a visual impairment due to optical atrophy since the beginning of his illness.

RADIOSURGICAL TECHNIQUE

After local anaesthetic scalp infiltration ( $1 \%$ xylocain), we screwed on to the patient's skull an MRI compatible Leksell stereotactic frame (Elekta Inst $\subset \mathrm{AB}$, Sweden). In all patients, T2 weighted and contrast enhanced $\mathrm{T} 1$ weighted multiplanar MRI with a slice thickness of $3 \mathrm{~mm}$ was performed. The target coordinates, the positioning of the isocentres, and the time of radiation was calculated using the KULA dose planning system (Elekta Inst $\mathbb{C} \mathrm{AB}$, Sweden). Radiosurgery was performed using a 201 source Cobalt 60 gamma knife unit (Elekta Inst $(\mathrm{AB}$, Sweden). The tumours in the lateral ventricle were treated with a marginal dose of 12.5 Gy to 16 Gy by enclosing the tumours with the $50 \%$ to $60 \%$ isodoseline. In one case, an additional tumour nodule at the bottom of the third ventricle was treated with 9.6 Gy only delivered to the $30 \%$ isodoseline by using small $4 \mathrm{~mm}$ isocentres with a steep fall off of irradiation, due to the tumour's proximity to the optical system. According to tumour size and the number of lesions 3, 12, and 17 isocentres were used (table 1). After GKRS, all three patients left hospital within 24 hours.

POSTRADIOSURGICAL EVALUATION

For the postradiosurgical follow up, as for the treatment planning, MRI with a slice thickness of $3 \mathrm{~mm}$ was chosen. The tumour volume at the time of treatment was calculated with the volumetric programme of our treatment planning system Gamma Plan 5. 12 (Elekta Inst (C) $\mathrm{AB}$, Sweden). For volumetry of the radiological follow up, the MR images were scanned and the volume was calculated using the Osiris medical imaging software developed at the University Hospital of Geneva, Switzerland. In the two cases of multifocal tumour recurrence, the volumes of all tumour nodules were added together.

\section{Results}

We encountered no radiosurgically related complications. After GKRS no patient devel-
Table 2 Changes in tumour volumes

\begin{tabular}{|c|c|c|c|}
\hline \multirow[b]{2}{*}{ Patient } & \multicolumn{3}{|l|}{ Volume } \\
\hline & $\begin{array}{l}\text { Pre-GKRS } \\
\left(\mathrm{mm}^{3}\right)\end{array}$ & $\begin{array}{l}\text { Post-GKRS } \\
\left(\mathrm{mm}^{3}\right)\end{array}$ & Shrinkage (\%) \\
\hline 1 & 600 & 250 (1 y) & 58 \\
\hline 2 & 5900 & $2300(2 \mathrm{y})$ & 61 \\
\hline 3 & 5200 & $3100(5 \mathrm{y})$ & 40 \\
\hline
\end{tabular}

GKRS=Gamma knife radiosurgery

oped new neurological deficits. One patient remained retired due to his pre-existing abducens palsy and visual impairment. The other two patients were integrated into their previous employment after GKRS. Unfortunately one of them died due to cardiac failure as a consequence of pericarditis 1 year after treatment. Therefore a clinical follow up as well as a neuroradiological follow up of 1,2 , and 5 years was achieved.

Tumour shrinkage was seen in all cases. The volume measured before radiosurgery ranged from $600 \mathrm{~cm}^{3}$ to $5900 \mathrm{~mm}^{3}$. Within the follow up period the volumes of the tumours in the lateral ventricles decreased and ranged from $250 \mathrm{~mm}^{3}$ to $3100 \mathrm{~mm}^{3}$. In one patient the tumour nodule in the third venticle had almost vanished by 2 years. Calculation of the percentile changes of the tumour volumes gave a decrease of $58 \%, 40 \%$, and $61 \%$ (table 2, figure).

\section{Discussion}

Central neurocytomas are characterised by a benign course, although malignant variants ${ }^{411-13}$ and disseminations have been reported..$^{11} 182024$ In line with the general characteristics of central neurocytomas, our patients were young adults, the onset of symptoms was abrupt, and all patients became symptomatic with signs of increased intracranial pressure. ${ }^{41415172526}$ The tumour locations in our series were mainly both lateral ventricles with extension to the third ventricle in one patient, such as described in previous reports. ${ }^{241316172527}$ The neuropathological investigation of the tumours showed no histological signs of malignancy and an expression of a typical neuronal antigenetic profile was detected in all three tumour specimens. Corresponding to the literature, all of the central neurocytomas were strongly positive for NSE and SYN. No immunohistochemical expression was found for NFP, GFAP, and S 100, exept in reactive astrocytic cells. $^{24121617192526}$ The MIB-1 proliferation index is reported to range from $0.1 \%$ to $8.6 \%,{ }^{32-30}$ and one author even reports an extremely high index of more than $50 \% .{ }^{11}$ The proliferation indices of our series were in the upper range with $2.4 \%, 7 \%$, and $8.7 \%$.

Microsurgical resection is the method of choice in primary treatment. ${ }^{14-17}$ Total tumour removal was reported in about half of the patients ${ }^{414151926}$ and tumour recurrences have also been reported. ${ }^{41214172526}$ Thus a strong demand remains for a safe adjunctive treatment of subtotally resected central neurocytomas but also of recurrence. Therapeutical policies include a "wait and see" strategy, 

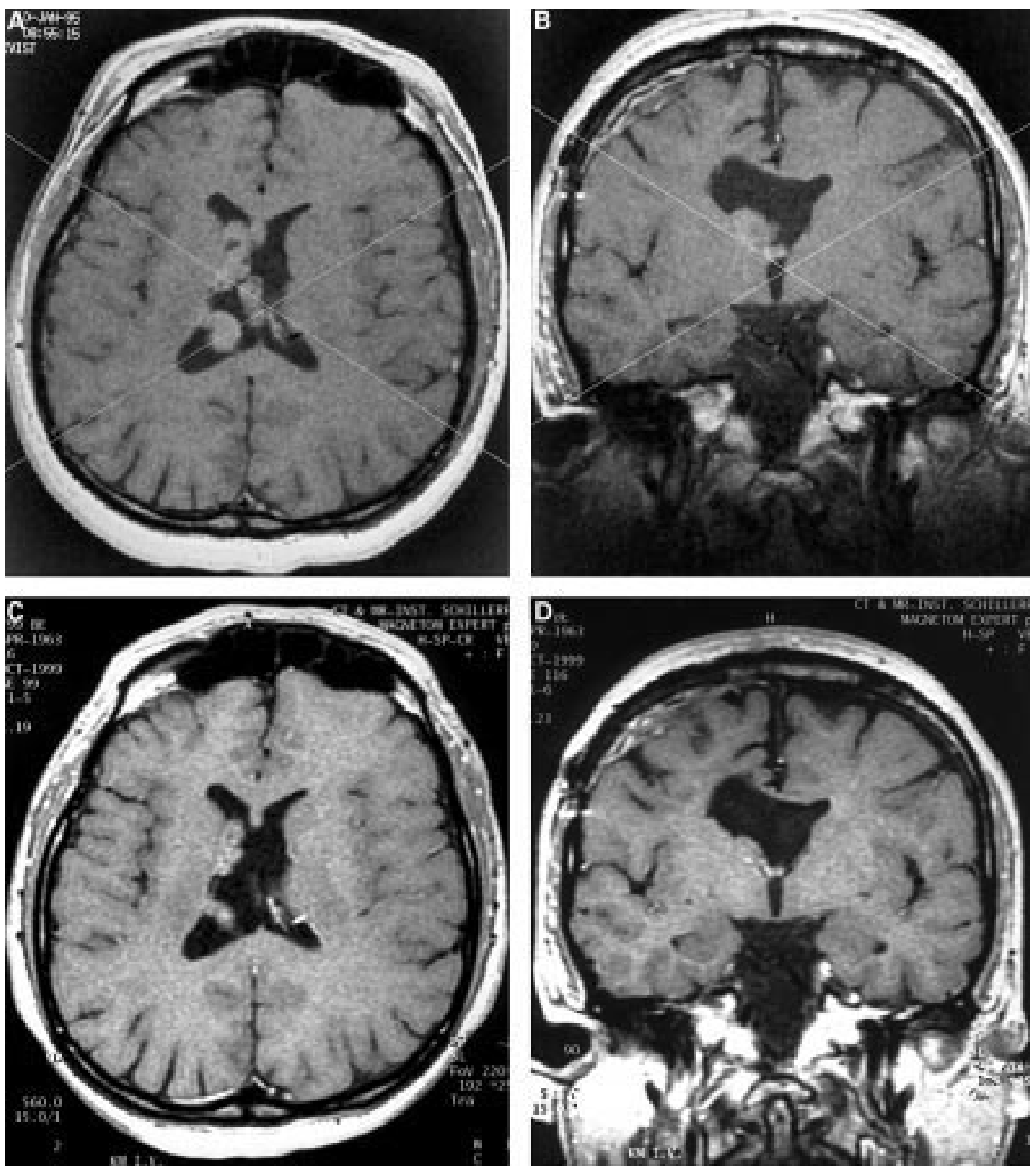

T1 weighted MRI after application of contrast medium shows the recurrent multilocal tumour nodules of a central neurocytoma $(C N)$ located in both lateral ventricles at the time of radiosurgery. (A) axial view; (B) coronal view treated with 13 Gy at the 60\% isodoseline. Five years after treatment, MRI demonstrates an obvious shrinkage of the total tumour mass. (C) axial view; (D) coronal view.

reoperation, chemotherapy, and radiation therapy. Only few reports have hitherto been published about the efficacy of chemotherapy, which is not widely used at present in combating this tumour entity. ${ }^{15} 1819$ The indication for radiation therapy is still controversial, although many authors performed radiotherapy after subtotal as well as after total resection. ${ }^{14-17} 1920$ Whole brain or local radiotherapy was applied by delivering a dose of 48 Gy to 61 Gy through 180 cGy to 200 cGy fractions ${ }^{14} 152028$ and radiological tumour shrinkage and even tumour disappearance has been described. ${ }^{14171920}$ Except for one case of demyelinating changes in the brain parenchyma adjacent to the tumour covered by the field of radiation therapy, there are no reports dealing with complications or undesired side effects of irradiation. ${ }^{14}$

Fujimaki et al reported on a patient in whom the MIB-1 proliferation activity after radiotherapy decreased from $5.6 \%$ to $0.25 \%{ }^{28}$ Schild et al reported a large series of 32 central neurocytomas followed up for 2.3 to 15.3 years. ${ }^{15}$ In their patients, total resection was achieved in 10 and subtotal resection in the remaining 22. Radiation therapy was administered to five patients with totally resected and to eight patients with subtotally resected central neurocytomas. They found that the 5 year local control rate and the 5 year survival rate could be improved by total resection as well as by radiation therapy. Kim et al described a series of 15 patients with central neurocytomas. ${ }^{14}$ Seven of them were totally resected, two of the patients received radiation therapy and had no recurrence within a follow up period of 41 to 94 months. Recurrences were found 8 and 21 months after surgery in two of the five remaining patients with totally resected, but not irradiated central neurocytomas. The tumours in the five patients who received radiation therapy after subtotal resection shrank or even disappeared during the follow up period of 27 to 113 months. Three patients who did not receive radiation therapy after 
subtotal resection had no change in their residual tumour after 4 to 76 months. Nakagawa et al compiled a review of the literature covering 26 patients who had received radiation therapy mainly after subtotal resection. ${ }^{20}$ They found three patients who had not received any further therapy, yet did well. In their own series radiation therapy caused tumour shrinkage and loss of contrast enhancement. Figarella et al reported a series of 20 central neurocytomas and recommended radiotherapy if total tumour removal was not possible. ${ }^{19}$ They also reported two patients with central neurocytomas treated by ventricle shunting, followed by radiation therapy only. Both patients did extremely well within a follow up period of 6 and 7 years.

There are currently no reports dealing specifically with GKRS as a means of controlling residual or recurrent central neurocytomas. Although Schild et al mentioned that one patient in their series of 13 irradiated central neurocytomas was treated with GKRS delivering a dose of $15 \mathrm{~Gy}$, they did not report the effects on that particular patient. ${ }^{15}$

In our series, multiple microsurgical resections via different approaches led to total removal. Total resection was confirmed by the surgical report and postoperative CT as MRI was not available at that time. Therefore, no additional radiation therapy was given. Nevertheless, new tumour growth occurred in all patients 5 to 6 years after open surgery. So surgery might have led to gross total resection only, if small residual tumours were not obvious on postoperative CT. The characteristics of tumour regrowth were the development of a single tumour nodule in the tumour bed of one patient and multilocal tumour nodules at different sites in the other two patients. On MRI these tumour nodules demonstrated moderate contrast enhancement and a clear demarcation from the surrounding brain tissue. In the literature no multicentric central neurocytoma recurrences have been reported so far. As these small nodules in both lateral ventricles and the third ventricle could not be resected, we chose GKRS for their treatment. For the follow up periods of 1 to 5 years the aim of GKRS - tumour control - was achieved in all cases. In addition, we found a significant decrease in tumour volume in all three recurrent central neurocytomas. All patients tolerated radiosurgery without presenting any additional neurological deficits.

In a slow growing tumour tissue with a low mitotic frequency as in central neurocytomas, the effect of radiosurgery is commonly thought to be primarily vascular damage to tumour vessels resulting in tumour necrosis. The other effect seems to be direct damage to the tumour tissue itself, as shown by histological examination. ${ }^{31}$ Central neurocytomas have good vascularisation; therefore they demonstrate a strong contrast enhancement. This vascularisation seems to be responsible for the excellent response of central neurocytomas to GKRS.

The advantage of a focused radiation is that a high dose with a steep fall off in radiation can be delivered precisely in one singe treatment session. In addition, the recurrent central neurocytomas are usually characterised by intraventricular growth, so they tend to be surrounded by CSF, and only a small part of the tumour has contact with the brain tissue. This makes them ideal targets for GKRS, as most of the dose surplus hits the CSF and the radiation burden to the neighbouring brain tissue can be kept at a minimum. In this way, the side effects of radiosurgery are minimised.

\section{Conclusion}

As recurrences of central neurocytomas have been reported after total resection with and without adjunctive radiotherapy, the use of external fractionated radiation therapy after total tumour resection remains questionable. We recommend yearly control MRI, which would make the detection of even small tumour recurrences possible. If these happen, GKRS allows open surgery to be avoided and is a safe and effective tool that can be administered within a single procedure. Thus, tumour control and even tumour shrinkage can be achieved with a low risk of side effects. To reach this goal, low doses of irradiation delivered to the tumour margin seem to be sufficient. As this is the first report on GKRS for central neurocytomas, a longer follow up and reports from other centres are required.

1 Hassoun J, Gambarelli D, Grisoli F, et al. Central neurocytoma: an electron-microscopic study of two cases. Acta toma: an electron-microscop

$2 \mathrm{Kim}$ DG, Chi JG, Sung SH, et al. Intraventricular neurocytoma: clinicopathological analysis of seven cases. $\mathcal{f}$ Neurosurg 1992;76:759-65.

3 Sharma MC, Rathore A, Karak AK, et al. A study of proliferative markers in central neurocytoma. Pathology 1998;30: 355-9.

4 Yasargil MG, von Ammon K, von Deimling A, et al. Central neurocytoma: histopathological variants and therapeutic approaches. F Neurosurg 1992;76:32-7.

5 Enam SA, Rosenblum ML, Ho KL. Neurocytoma in the cerebellum: case report. F Neurosurg 1997;87:100-2.

6 Fukui M, Matsushima T, Fujii S, et al. Pineal and third ventricle tumours in the CT and MR eras. Acta Neurochir Suppl (Wien) 1991;53:127-36.

7 Roche PH, Malca S, Gambarelli D, et al. Giant central neuRoche $\mathrm{PH}$, Malca S, Gambarelli D, et al. Giant central neu-
rocytoma with tetraventricular and extra-axial extension: rocytoma with tetraventricular and extra-axial exten

8 Sgouros S, Jackowski A, Carey M. Central neurocytoma Sgouros S, Jackowski A, Carey M. Central neurocytoma
without intraventricular extension. Surg Neurol 1994;42:

without intraventricular extension. Surg Neurol 1994;42:
$335-9$.
Sgouros S, Walsh AR, Barber P. Central neurocytoma of thalamic origin. Br $\mathcal{F}$ Neurosurg 1994;8:373-6.

10 Tattler SB, Borges LF, Louis DN. Central neurocytomas of the cervical spinal cord: report of two casse. 7 Neurosurg 1994;81:288-93

11 Jay V, Edwards V, Hoving E, et al. Central neurocytoma: morphological, flow cytrometric, polimerase chain reaction, fluorescence in situ hybridization, and kariotypic analysis. F Neurosurg 1999;90:348-54

12 von Deimling A, Janzer R, Kleihues P, et al. Patterns of differentiation in central neurocytoma: an immunhistochemical study of 11 biopsies. Acta Neuropathol 1990;79:473-9.

13 Wiechmann W, Schubiger O, von Deimling A, et al. Neuroradiology of central neurocytoma. Neuroradiology 1991;33: radiology.

14 Kim DG, Paek SH, Kim IH, et al. Central neurocytoma: the role of radiation therapy and long term outcome. Cancer 1997;79:1995-2002.

15 Schild SE, Scheithauer BW, Haddock MG, et al. Central neurocytomas. Cancer 1997;79:790-5.

16 Tacconi L, Thom M, Symon L. Central neurocytoma: a clinico-pathological study of five cases. $\mathrm{Br} \mathcal{F}$ Neurosurg 1997;11:286-91.

17 Valdueza JM, Westphal M, Vortmeyer A, et al. Central neurocytoma: clinical, immunohistologic, and biologic findings of a human neuroglial progenitor tumor. Surg Neurol 1996;45:49-56.

18 Eng DY, DeMonte F, Ginsberg L, et al. Craniospinal dissemination of central neurocytoma. 7 Neurosurg 1997; 86:547-52.

19 Figarella-Branger D, Pelissier JF, Daumas-Duport C, et al. Central neurocytomas. Critical evaluation of a small-cel neuronal tumor. Am $\mathcal{f}$ Surg Pathol 1992;16:97-109. 
20 Nakagawa K, Aoki Y, Sakata K, et al. Radiation therapy of well-differentiated neuroblastoma and central neurocytoma. Cancer 1993:72:1350-5.

21 Leksell DG. Special stereotactic techniques: stereotactic radiosurgery. In: Heilbrun MP, ed. Stereotactic neurosurgery. Baltimore: Williams and Wilkins 1988:195-209.

22 Lunsford LD, Flickinger J, Lindner G, et al. Stereotactic radiosurgery of the brain using the first United States 201 cobald 60 source $\gamma$ knife. Neurosurgery 1989;24:151-9

23 Kondziolka D, Lunsford LD, McLaughlin MR, et al. Long term outcomes after radiosurgery for acoustic neurinomas. N Engl f Med 1998;339:1426-33

24 Yamamoto T, Komori T, Shibata N, et al. Multifocal neurocytoma / gangliocytoma with extensive leptomeningeal dissemination in the brain and spinal cord. Am f Surg Pathol 1996;20:363-70.

25 Kim DG, Kim JS, Chi JG, et al. Central neurocytoma: proliferative potential and biological behavior. $\mathcal{f}$ Neurosurg 1996;84:742-7.
26 Nishio S, Tashima T, Takeshita I, et al. Intraventricular neurocytoma: clinicopathological features of six cases. $\mathcal{F}$ Neurosurg 1988;68:665-70.

27 Goergen SK, Gonzales MF, McLean CA. Intraventricular neurocytoma: radiologic features and review of the literature. Radiology 1992;182:787-92.

28 Fujimaki T, Matsuno A, Sasaki T, et al. Proliferative activity of central neurocytoma: measurement of tumor volume doubling time, MIB-1 staining index and bromodeoxyuridine labeling index. F Neurooncol 1997;32:103-9.

29 Mackenzie IR. Central neurocytoma: histologic atypia, proiferation potential, and clinical outcome. Cancer 1999;85. $1606-10$.

30 Soylemezoglu F, Scheithauer BW, Esteve J, et al. Atypical central neurocytoma. F Neuropathol Exp Neurol 1997;56: $551-6$.

31 Noren G, Arndt J, Hindmarsh T. Stereotactic radiosurgery in cases of acoustic neurinoma: further experiences. Neurosurgery $1983 ; 13: 12-22$.

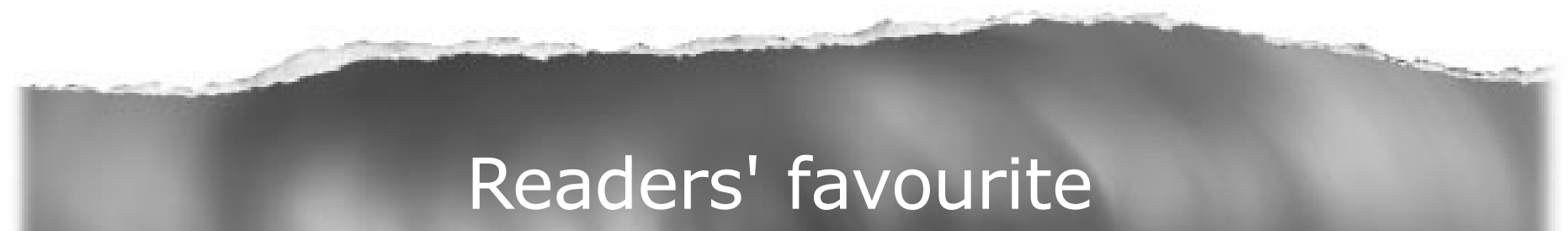

Top 10

Click on the "Top 10" button on the homepage to see which are the best read articles each month

www.jnnp.com 